\title{
EQ-5D-5L norms for the urban Chinese population in China
}

\author{
Zhihao Yang ${ }^{1,2^{*}}$ (D) Jan Busschbach ${ }^{2}$, Gordon $\mathrm{Liu}^{3}$ and Nan Luo ${ }^{4}$
}

\begin{abstract}
Background: To generate Chinese population norms for the EQ-5D-5L dimensions, EQ-VAS (Visual Analogue Scale) scores and EQ-5D-5L index scores, stratified by gender and age. The EQ-5D is a widely used generic health-related quality of life instrument to describe population health and health outcomes in clinical trials and health economic evaluations. Currently, there are no EQ-5D-5L population norms for China.

Methods: This norm study utilized the data collected in an EQ-5D-5L valuation study in China between December 2012 and January 2013. In the valuation study, respondents were asked to report their own health states using the EQ-5D-5L descriptive system and the EQ-VAS. Respondents' demographic information was also collected. The EQ index score was calculated using the EQ-5D-5L value set based on the Chinese urban population. Norm scores were reported by important demographic variables.
\end{abstract}

Results: The mean EQ-VAS scores ranged between 88.3 for males of $<19$ years and 82.9 for females of 60-69 years. Contrary to other population studies, females reported higher EQ-VAS scores than males in every age group except for 20-29 years. The mean EQ-5D-5L index values ranged from 0.912 for females of $>70$ years to 0.971 for females of 30-39 years. Respondents reported more problems in the dimensions 'pain/discomfort' and 'anxiety/depression' than in the dimensions 'mobility', 'self-care' and 'usual activities' in all age groups.

Conclusions: The population norm scores for the EQ-5D can be used as reference values for comparative purposes in future Chinese studies. Further research into rural and/or a more representative population is warranted.

Keywords: EQ-5D-5L, Population norm, China, HRQoL

\section{Introduction}

EQ-5D is a health-related quality of life (HRQoL) questionnaire widely used in economic, clinical, and population health studies. The EQ-5D descriptive system comprises five dimensions: mobility, self-care, usual activities, pain/discomfort and anxiety/depression [1]. It has two versions, a three-level EQ-5D (EQ-5D-3L) and a five-level EQ-5D (EQ-5D-5L). Although EQ-5D-3L has been widely used, it is reported to suffer from ceiling effects (i.e. the percentage of respondent reporting the best possible health state on EQ-5D) and measurement insensitivity [2]. By increasing the number of levels in the descriptive system,

\footnotetext{
*Correspondence: zhihao_yang_cn@126.com

'Health Services Management Department, Guizhou Medical University, No.9 Beijing Road, Guiyang, China

${ }^{2}$ Medical Psychology and Psychotherapy, Erasmus Medical Center,

Wytemaweg, 80 Rotterdam, The Netherlands

Full list of author information is available at the end of the article
}

EQ-5D-5L has demonstrated reduced ceiling effects and improved discriminatory power in comparison to EQ-5D-3L [3-7]. In addition to classifying health states in terms of the 5 dimensions of health, EQ-5D permits the valuation of these health states. This is accomplished from both the respondent's own perspective by using a Visual Analogue Scale (EQ-VAS) and from the perspective of the general public's by attaching the appropriate EQ-5D index score to the described health state of the respondent.

EQ-5D has been used to measure population health in many countries, and population norms have been established by age, gender and socio-economic status [1]. A set of population norm scores provides an important reference point for clinical and health economic research outcomes, as the effects of medical conditions and/or treatments can be quantified by comparing patients and/or intervention groups with the general population [8]. At this juncture,

(c) The Author(s). 2018 Open Access This article is distributed under the terms of the Creative Commons Attribution 4.0 International License (http://creativecommons.org/licenses/by/4.0/), which permits unrestricted use, distribution, and reproduction in any medium, provided you give appropriate credit to the original author(s) and the source, provide a link to the Creative Commons license, and indicate if changes were made. The Creative Commons Public Domain Dedication waiver (http://creativecommons.org/publicdomain/zero/1.0/) applies to the data made available in this article, unless otherwise stated. 
there are no EQ-5D-5L norms for the Chinese population, which hampers the increasing use of EQ-5D-5L in China.

The objective of this paper is to provide population norm, including the prevalence of EQ-5D-5L health problems, and EQ-VAS and EQ index scores by age and gender, in the Chinese urban population. In addition, we also examine the relationships between socio-economic factors and (i) the components of the EQ-5D-5L descriptive system, (ii) EQ-VAS scores and (iii) EQ index scores.

\section{Methods}

\section{Sampling and recruitment}

This norm study drew data from a large EQ-5D-5L valuation study in China [3]. In the valuation study, respondents reported: 1) valuation data and; 2) self-reported health status data and demographic data. The valuation data was used to establish the EQ-5D-5L value set for China and was reported elsewhere [3]. This study used the self-reported health status data and demographic data to establish a population norm. The sample size was decided by the EQ-5D-5L valuation protocol, which was aiming at constructing country specific EQ-5D-5L value set [9]. Members of the general population were randomly recruited from five urban areas of five cities (Beijing, Shenyang, Nanjing, Chengdu, and Guiyang). From each city, respondents were recruited from at least five difference administrative districts and at different time of day. Specific recruitment sites included library, hospital, university, local community, park and shopping areas etc. [10]. These five cities were selected as representative urban areas in terms of size of population, geographical region and economic development status in China [3]. Within each city, quotas were set to recruit equal numbers of participants from each city and to ensure the study sample resembled the general Chinese urban adult population with respect to age, gender, and education level according to the Sixth National Population Census [10]. In each city, members of the general public who were at least 16 years old, and were literate and able to understand survey questions, were recruited through personal invitation [3]. Response rate was calculated.

Each respondent was interviewed face-to-face by a trained interviewer using the EuroQol valuation technology (EQ-VT) $[3,11]$. EQ-VT is a standardized software design by the EuroQol Group in order to facilitate the data collection for valuation study [9]. The interview had four sections. The first section was for respondents to report their own health using the EQ-5D-5L questionnaire: the five-dimensional descriptive system and the EQ-VAS. In the second section respondents were asked to value 10 different EQ-5D-5L health states using a composite time trade-off (cTTO) method [8]. The third section contained 7 pairs of
EQ-5D-5L discrete choice tasks. The fourth section assessed respondents' socio-economic and other background characteristics. This paper used data collected in the first and fourth sections only.

\section{The EQ-5D questionnaire}

The EQ-5D-5L descriptive system consists of five dimensions (mobility, self-care, usual activities, pain/ discomfort and anxiety/depression) with five ordinal severity levels each (no problems, slight problems, moderate problems, severe problems, and extreme problems/unable to), thus defining $3125\left(5^{5}\right)$ distinct health states [2]. The respondent is asked to indicate his/her health state against the most appropriate statement in each of the 5 dimensions and this leads to a 1-digit number expressing the level selected for each dimension [1], i.e. 12211 means the respondent had no problems in mobility, pain/discomfort, and anxiety/depression, but had slight problems in self-care and usual activities. A VAS was used in the interview, with anchor points 0 ('worst imaginable health state') and 100 ('best imaginable health state'). Respondents first report their own health state using the EQ-5D-5L descriptive system and then their overall health on the EQ-VAS based on their health on the day of survey.

In 2012, the Chinese version of EQ-5D-5L was translated using a response scaling method by Luo et al. [2].The translation process followed the standard translation protocol developed by the EuroQol Group and had mainly 3 stages [2]. First, different candidate labels were generated by direct translation and reviewing existing questionnaire; second, 50 native Chinese speaking respondents were interviewed to rank and value potential candidates labels; third, the final labels were selected to achieve comparable measurement properties to the English/Spanish version of EQ-5D-5L. The translated Chinese EQ-5D-5L demonstrated validity and increased sensitivity in diabetes and hepatitis $\mathrm{B}$ patients [12, 13].

\section{Data analysis}

For each respondent, the EQ-5D-5L health state and the EQ-VAS were directly observed from respondent's' self-report questionnaire while the $\mathrm{EQ}$ index score was derived from the Chinese EQ-5D-5L value set [3]. In the EQ-5D-5L value set, the EQ index score of all 3125 health states were estimated [3]. For each respondent, we derived their corresponding EQ index score from their self-reported health states.

First, descriptive statistics of EQ-5D-5L health state, EQ-VAS and EQ index score were calculated for the whole sample and by different demographic variables and cities (age, gender, employment status etc.). For comparison, we categorized age into age groups following other 
countries' population norm studies [6, 14-17]. As our sample was recruited from urban areas only, we used 'Residence of origin' as a proxy to study the possible difference of HRQoL between urban and rural residents. 'Residence of origin' referred to the birth place of the respondent and was classified into three groups (city, county, township or village) based on China's administrative levels. 'Health insurance' referred to social health insurance types in China's healthcare system [18]. For each demographic variable, the percentage of reported problem in EQ-5D dimension, the means (and 95\% confidence interval) of EQ-VAS and EQ index scores were calculated for each subgroup and the difference were tested statistically. Second, we used multivariable analysis to examine the associations between demographic characteristics with reported problems in EQ-5D-5L, EQ-VAS and EQ index scores respectively. For the reported problems in each dimension, we used logistic regression ('no problems' coded as 0; 'slight problems', 'moderate problems', 'severe problems', or 'extreme problems/unable' coded as 1) [1]. For EQ-VAS and EQ index scores, we used linear regression. All demographic variables including age and education level were entered into the models as categorical variables. For better interpretation, age groups were collapsed into three categories (young age: $<=29$ years, middle age: 30 59 years; older age: $>=60$ years) [14]. Multivariable analysis was used to identify significant demographic characteristics using a backward selection procedure to remove covariates with $p>0.05$. Odds ratio was reported for logistic regression and coefficient was reported for linear regression respectively, the corresponding $95 \% \mathrm{CI}$ was calculated using robust standard error.

For this study, ethical approval was not needed in China at the time of data collection. A waiver of the informed consent was approved as this study did not provide any intervention to participants. Participants can withdraw at any time without any consequences.

\section{Results}

A total of 1332 individuals (response rate: 68.6\%) who met the inclusion criteria were recruited. Among these, 1296 (97.3\%) who successfully completed the questionnaire were included in the analysis. The mean age of the sample was 42 years (SD: 16 years), the age ranged between 16 years to 85 years old. Females comprised $49.9 \%$ of the sample. Other demographic information is shown in Table 1.

In total, $54 \%$ of the sample reported their health as '11111', followed by '11121', '11112', '11122', and '21121'. The percentages of 'no problems' were: $94.37 \%$ for mobility, $98.92 \%$ for self-care, $95.45 \%$ for usual activity, $70.14 \%$ for pain/discomfort, and $73.15 \%$ for anxiety/depression. The mean EQ-VAS and EQ index scores were 86.0 (SD: 11.4 ) and 0.957 (SD: 0.069), respectively.
Table 1 Demographic characteristics of all respondents

\begin{tabular}{|c|c|c|}
\hline \multirow[b]{2}{*}{ Age group, years } & \multicolumn{2}{|c|}{ Our sample } \\
\hline & $N$ & $\%$ \\
\hline $16-19$ & 109 & 8.4 \\
\hline $20-29$ & 229 & 17.7 \\
\hline $30-39$ & 244 & 18.8 \\
\hline $40-49$ & 272 & 21.0 \\
\hline $50-59$ & 220 & 17.0 \\
\hline $60-69$ & 155 & 12.0 \\
\hline$>70$ & 67 & 5.2 \\
\hline \multicolumn{3}{|l|}{ Gender } \\
\hline Female & 646 & 49.9 \\
\hline Male & 650 & 50.2 \\
\hline \multicolumn{3}{|l|}{ Education } \\
\hline Primary or lower & 138 & 10.7 \\
\hline Junior \& Senior high school & 867 & 66.9 \\
\hline College or higher & 291 & 22.5 \\
\hline \multicolumn{3}{|l|}{ Employment status } \\
\hline Full time employees & 382 & 29.5 \\
\hline Temporary worker \& freelancer & 451 & 34.8 \\
\hline Retired & 240 & 18.5 \\
\hline Student & 132 & 10.2 \\
\hline Other & 91 & 7.0 \\
\hline \multicolumn{3}{|l|}{ Residence of origin } \\
\hline City & 757 & 58.4 \\
\hline County & 86 & 6.6 \\
\hline Township or village & 453 & 35.0 \\
\hline \multicolumn{3}{|l|}{ Health insurance } \\
\hline Urban employee & 551 & 42.5 \\
\hline Urban residence & 304 & 23.5 \\
\hline New rural & 296 & 22.8 \\
\hline Other & 88 & 6.8 \\
\hline No & 57 & 4.4 \\
\hline
\end{tabular}

Tables 2 and 3 show the percentage of reported problems for each severity level and EQ-5D dimension, and the mean (SD) of EQ-VAS and EQ index scores for males and females by age groups, respectively. In both male and female groups, the number of problems increased with age in the dimensions of mobility, self-care, and pain/discomfort $(p<0.05$, trend test for ordered groups). In contrast, anxiety/ depression was more prevalent in younger age groups $(p<0.01$, trend test for ordered groups). As could be expected, the means of both EQ-VAS and EQ index scores decreased with age, but only the EQ index score for male was statistically significant $(p<0.05$, trend test for ordered groups). Females reported 
Table 2 Percentage of a general population sample reporting levels 1 to 5 by dimension, EQ-VAS \& EQ index score by age group for males

\begin{tabular}{|c|c|c|c|c|c|c|c|c|c|}
\hline \multirow[t]{2}{*}{ EQ-5D dimension } & & \multicolumn{7}{|c|}{ Age Groups } & \multirow{2}{*}{$\begin{array}{l}\text { Total } \\
N=650\end{array}$} \\
\hline & & $\begin{array}{l}16-19 \\
N=56\end{array}$ & $\begin{array}{l}20-29 \\
N=116\end{array}$ & $\begin{array}{l}30-39 \\
N=123\end{array}$ & $\begin{array}{l}40-49 \\
N=135\end{array}$ & $\begin{array}{l}50-59 \\
N=110\end{array}$ & $\begin{array}{c}60-69 \\
N=84\end{array}$ & $\begin{array}{l}>70 \\
N=26\end{array}$ & \\
\hline \multirow[t]{6}{*}{ Mobility } & No problems & $100 \%$ & $98.3 \%$ & $98.4 \%$ & $91.9 \%$ & $96.4 \%$ & $85.7 \%$ & $69.2 \%$ & $94.0 \%$ \\
\hline & Slight problems & $0 \%$ & $1.7 \%$ & $1.6 \%$ & $8.2 \%$ & $3.6 \%$ & $13.1 \%$ & $26.9 \%$ & $5.7 \%$ \\
\hline & Moderate problems & $0 \%$ & $0 \%$ & $0 \%$ & $0 \%$ & $0 \%$ & $1.2 \%$ & $3.9 \%$ & $0.3 \%$ \\
\hline & Severe problems & $0 \%$ & $0 \%$ & $0 \%$ & $0 \%$ & $0 \%$ & $0 \%$ & $0 \%$ & $0 \%$ \\
\hline & Unable to & $0 \%$ & $0 \%$ & $0 \%$ & $0 \%$ & $0 \%$ & $0 \%$ & $0 \%$ & $0 \%$ \\
\hline & $Z$ ( $P$ value) & & & & & & & $5.69(0.0$ & \\
\hline \multirow[t]{6}{*}{ Self-care } & No problems & $100 \%$ & $100 \%$ & $100 \%$ & $98.5 \%$ & $100 \%$ & $96.4 \%$ & $96.2 \%$ & $99.1 \%$ \\
\hline & Slight problems & $0 \%$ & $0 \%$ & $0 \%$ & $1.5 \%$ & $0 \%$ & $3.6 \%$ & $3.9 \%$ & $0.9 \%$ \\
\hline & Moderate problems & $0 \%$ & $0 \%$ & $0 \%$ & $0 \%$ & $0 \%$ & $0 \%$ & $0 \%$ & $0 \%$ \\
\hline & Severe problems & $0 \%$ & $0 \%$ & $0 \%$ & $0 \%$ & $0 \%$ & $0 \%$ & $0 \%$ & $0 \%$ \\
\hline & Unable to & $0 \%$ & $0 \%$ & $0 \%$ & $0 \%$ & $0 \%$ & $0 \%$ & $0 \%$ & $0 \%$ \\
\hline & Z (P value) & & & & & & & $2.65(0.0$ & \\
\hline \multirow[t]{6}{*}{ Usual Activity } & No problems & $96.4 \%$ & $94.8 \%$ & $95.9 \%$ & $93.3 \%$ & $99.1 \%$ & $90.5 \%$ & $92.3 \%$ & $94.9 \%$ \\
\hline & Slight problems & $3.6 \%$ & $5.2 \%$ & $4.1 \%$ & $5.9 \%$ & $0.9 \%$ & $7.1 \%$ & $7.7 \%$ & $4.6 \%$ \\
\hline & Moderate problems & $0 \%$ & $0 \%$ & $0 \%$ & $0.7 \%$ & $0 \%$ & $2.4 \%$ & $0 \%$ & $0.5 \%$ \\
\hline & Severe problems & $0 \%$ & $0 \%$ & $0 \%$ & $0 \%$ & $0 \%$ & $0 \%$ & $0 \%$ & $0 \%$ \\
\hline & Unable to & $0 \%$ & $0 \%$ & $0 \%$ & $0 \%$ & $0 \%$ & $0 \%$ & $0 \%$ & $0 \%$ \\
\hline & Z (P value) & & & & & & & $0.95(0.3$ & \\
\hline \multirow[t]{6}{*}{ Pain/Discomfort } & No problems & $78.6 \%$ & $75.9 \%$ & $78.1 \%$ & $71.1 \%$ & $64.6 \%$ & $64.3 \%$ & $57.7 \%$ & $71.4 \%$ \\
\hline & Slight problems & $19.6 \%$ & $23.3 \%$ & $20.3 \%$ & $26.7 \%$ & $29.1 \%$ & $31.0 \%$ & $30.8 \%$ & $25.4 \%$ \\
\hline & Moderate problems & $1.8 \%$ & $0 \%$ & $0.8 \%$ & $1.5 \%$ & $6.4 \%$ & $4.8 \%$ & $11.5 \%$ & $2.8 \%$ \\
\hline & Severe problems & $0 \%$ & $0.9 \%$ & $0.8 \%$ & $0.7 \%$ & $0 \%$ & $0 \%$ & $0 \%$ & $0.5 \%$ \\
\hline & Extreme problems & $0 \%$ & $0 \%$ & $0 \%$ & $0 \%$ & $0 \%$ & $0 \%$ & $0 \%$ & $0 \%$ \\
\hline & Z (P value) & & & & & & & $3.44(0.0$ & \\
\hline \multirow[t]{6}{*}{ Anxiety/Depression } & No problems & $67.9 \%$ & $65.5 \%$ & $66.7 \%$ & $78.5 \%$ & $75.5 \%$ & $77.4 \%$ & $88.5 \%$ & $72.8 \%$ \\
\hline & Slight problems & $30.4 \%$ & $32.8 \%$ & $29.3 \%$ & $20.7 \%$ & $21.8 \%$ & $20.2 \%$ & $11.5 \%$ & $25.1 \%$ \\
\hline & Moderate problems & $1.8 \%$ & $1.7 \%$ & $2.4 \%$ & $0 \%$ & $1.8 \%$ & $0 \%$ & $0 \%$ & $1.2 \%$ \\
\hline & Severe problems & $0 \%$ & $0 \%$ & $0.8 \%$ & $0.7 \%$ & $0.9 \%$ & $2.4 \%$ & $0 \%$ & $0.6 \%$ \\
\hline & Extreme problems & $0 \%$ & $0 \%$ & $0.8 \%$ & $0 \%$ & $0 \%$ & $0 \%$ & $0 \%$ & $0.3 \%$ \\
\hline & Z (P value) & & & & & & & $-2.94(0$ & \\
\hline \multirow[t]{4}{*}{ EQ-VAS } & Mean & 87.4 & 86.9 & 85.5 & 85.5 & 84.8 & 82.9 & 83.9 & 85.4 \\
\hline & $95 \% \mathrm{Cl}$ & 84.4 & 85.2 & 83.8 & 83.3 & 82.6 & 79.9 & 76.9 & 84.5 \\
\hline & & 90.4 & 88.5 & 87.2 & 87.8 & 87.1 & 85.9 & 90.9 & 86.3 \\
\hline & Z (P value) & & & & & & & $-1.68(0$ & \\
\hline \multirow[t]{4}{*}{ EQ index score } & Mean & 0.968 & 0.963 & 0.961 & 0.959 & 0.956 & 0.943 & 0.932 & 0.957 \\
\hline & $95 \% \mathrm{Cl}$ & 0.957 & 0.953 & 0.950 & 0.948 & 0.946 & 0.921 & 0.897 & 0.952 \\
\hline & & 0.978 & 0.973 & 0.972 & 0.971 & 0.967 & 0.964 & 0.966 & 0.962 \\
\hline & Z (P value) & & & & & & & \multicolumn{2}{|c|}{$-2.21(0.027)$} \\
\hline
\end{tabular}

higher EQ-VAS values than males $(\mathrm{p}<0.05$, two-sample t-test). The highest mean EQ index score was observed for females of 30-39 years (0.971), the lowest mean score for females of $>70$ years (0.912). The mean VAS score ranged between 88.3 for females of $<19$ years and 82.9 for males of 60-69 years. 
Table 3 Percentage of a general population sample reporting levels 1 to 5 by dimension, EQ-VAS \& EQ index score by age group for females

\begin{tabular}{|c|c|c|c|c|c|c|c|c|c|}
\hline \multirow[t]{2}{*}{ EQ-5D dimension } & & \multicolumn{7}{|c|}{ Age Groups } & \multirow{2}{*}{$\begin{array}{l}\text { Total } \\
N=646\end{array}$} \\
\hline & & $\begin{array}{l}16-19 \\
N=53\end{array}$ & $\begin{array}{l}20-29 \\
N=113\end{array}$ & $\begin{array}{l}30-39 \\
N=121\end{array}$ & $\begin{array}{l}40-49 \\
N=137\end{array}$ & $\begin{array}{l}50-59 \\
N=110\end{array}$ & $\begin{array}{c}60-69 \\
N=71\end{array}$ & $\begin{array}{l}>=70 \\
N=41\end{array}$ & \\
\hline \multirow[t]{6}{*}{ Mobility } & No problems & $96.2 \%$ & $96.5 \%$ & $99.2 \%$ & $97.1 \%$ & $95.5 \%$ & $90.1 \%$ & $73.2 \%$ & $94.7 \%$ \\
\hline & Slight problems & $3.8 \%$ & $3.5 \%$ & $0.8 \%$ & $2.9 \%$ & $3.6 \%$ & $8.5 \%$ & $19.5 \%$ & $4.5 \%$ \\
\hline & Moderate problems & $0 \%$ & $0 \%$ & $0 \%$ & $0 \%$ & $0 \%$ & $1.4 \%$ & $7.3 \%$ & $0.6 \%$ \\
\hline & Severe problems & $0 \%$ & $0 \%$ & $0 \%$ & $0 \%$ & $0.9 \%$ & $0 \%$ & $0 \%$ & $0.2 \%$ \\
\hline & Unable to & $0 \%$ & $0 \%$ & $0 \%$ & $0 \%$ & $0 \%$ & $0 \%$ & $0 \%$ & $0 \%$ \\
\hline & Z (P value) & & & & & & & $4.68(0.0$ & \\
\hline \multirow[t]{6}{*}{ Self-care } & No problems & $98.1 \%$ & $99.1 \%$ & $99.2 \%$ & $100 \%$ & $99.1 \%$ & $97.2 \%$ & $95.1 \%$ & $98.8 \%$ \\
\hline & Slight problems & $1.9 \%$ & $0.9 \%$ & $0.8 \%$ & $0 \%$ & $0 \%$ & $1.4 \%$ & $4.9 \%$ & $0.9 \%$ \\
\hline & Moderate problems & $0 \%$ & $0 \%$ & $0 \%$ & $0 \%$ & $0 \%$ & $1.4 \%$ & $0 \%$ & $0.2 \%$ \\
\hline & Severe problems & $0 \%$ & $0 \%$ & $0 \%$ & $0 \%$ & $0.9 \%$ & $0 \%$ & $0 \%$ & $0.2 \%$ \\
\hline & Unable to & $0 \%$ & $0 \%$ & $0 \%$ & $0 \%$ & $0 \%$ & $0 \%$ & $0 \%$ & $0 \%$ \\
\hline & Z (P value) & & & & & & & $1.42(0.1$ & \\
\hline \multirow[t]{6}{*}{ Usual Activity } & No problems & $96.2 \%$ & $99.1 \%$ & $98.4 \%$ & $97.8 \%$ & $96.4 \%$ & $93.0 \%$ & $78.1 \%$ & $96.0 \%$ \\
\hline & Slight problems & $3.8 \%$ & $0.9 \%$ & $1.7 \%$ & $2.2 \%$ & $1.8 \%$ & $7.0 \%$ & $22.0 \%$ & $3.7 \%$ \\
\hline & Moderate problems & $0 \%$ & $0 \%$ & $0 \%$ & $0 \%$ & $0.9 \%$ & $0 \%$ & $0 \%$ & $0.2 \%$ \\
\hline & Severe problems & $0 \%$ & $0 \%$ & $0 \%$ & $0 \%$ & $0.9 \%$ & $0 \%$ & $0 \%$ & $0.2 \%$ \\
\hline & Unable to & $0 \%$ & $0 \%$ & $0 \%$ & $0 \%$ & $0 \%$ & $0 \%$ & $0 \%$ & $0 \%$ \\
\hline & Z (P value) & & & & & & & $4.36(0.0$ & \\
\hline \multirow[t]{6}{*}{ Pain/Discomfort } & No problems & $66.0 \%$ & $74.3 \%$ & $76.0 \%$ & $69.3 \%$ & $65.5 \%$ & $64.8 \%$ & $51.2 \%$ & $68.9 \%$ \\
\hline & Slight problems & $30.2 \%$ & $24.8 \%$ & $23.1 \%$ & $28.5 \%$ & $32.7 \%$ & $32.4 \%$ & $39.0 \%$ & $28.8 \%$ \\
\hline & Moderate problems & $1.9 \%$ & $0.9 \%$ & $0.8 \%$ & $1.5 \%$ & $0.9 \%$ & $2.8 \%$ & $7.3 \%$ & $1.7 \%$ \\
\hline & Severe problems & $1.9 \%$ & $0 \%$ & $0 \%$ & $0.7 \%$ & $0.9 \%$ & $0 \%$ & $2.4 \%$ & $0.5 \%$ \\
\hline & Extreme problems & $0 \%$ & $0 \%$ & $0 \%$ & $0 \%$ & $0 \%$ & $0 \%$ & $0 \%$ & $0.2 \%$ \\
\hline & Z (P value) & & & & & & & $2.56(0.0$ & \\
\hline \multirow[t]{6}{*}{ Anxiety/Depression } & No problems & $56.6 \%$ & $62.8 \%$ & $76.9 \%$ & $75.9 \%$ & $76.4 \%$ & $85.9 \%$ & $78.1 \%$ & $73.5 \%$ \\
\hline & Slight problems & $37.7 \%$ & $31.9 \%$ & $20.7 \%$ & $21.9 \%$ & $21.8 \%$ & $14.1 \%$ & $19.5 \%$ & $23.7 \%$ \\
\hline & Moderate problems & $5.7 \%$ & $4.4 \%$ & $2.5 \%$ & $1.5 \%$ & $1.8 \%$ & $0 \%$ & $2.4 \%$ & $2.5 \%$ \\
\hline & Severe problems & $0 \%$ & $0.9 \%$ & $0 \%$ & $0 \%$ & $0 \%$ & $0 \%$ & $0 \%$ & $0.2 \%$ \\
\hline & Extreme problems & $0 \%$ & $0 \%$ & $0 \%$ & $0.7 \%$ & $0 \%$ & $0 \%$ & $0 \%$ & $0.2 \%$ \\
\hline & Z (P value) & & & & & & & $-4.02(0$ & \\
\hline \multirow[t]{3}{*}{ EQ-VAS } & Mean & 88.3 & 85.8 & 87.8 & 87.5 & 86.2 & 84.5 & 85.3 & 86.6 \\
\hline & $95 \% \mathrm{Cl}$ & $\begin{array}{l}85.4 \\
91.2\end{array}$ & $\begin{array}{l}83.6 \\
88.0\end{array}$ & $\begin{array}{l}86.0 \\
89.6\end{array}$ & $\begin{array}{l}85.6 \\
89.3\end{array}$ & $\begin{array}{l}84.0 \\
88.3\end{array}$ & $\begin{array}{l}81.8 \\
87.2\end{array}$ & $\begin{array}{l}82.0 \\
88.6\end{array}$ & $\begin{array}{l}85.8 \\
87.5\end{array}$ \\
\hline & Z (P value) & & & & & & & $-1.75(0$ & \\
\hline \multirow[t]{3}{*}{ EQ index score } & Mean & 0.945 & 0.959 & 0.971 & 0.962 & 0.954 & 0.957 & 0.912 & 0.957 \\
\hline & $95 \% \mathrm{Cl}$ & $\begin{array}{l}0.926 \\
0.963\end{array}$ & $\begin{array}{l}0.949 \\
0.968\end{array}$ & $\begin{array}{l}0.962 \\
0.979\end{array}$ & $\begin{array}{l}0.952 \\
0.972\end{array}$ & $\begin{array}{l}0.933 \\
0.975\end{array}$ & $\begin{array}{l}0.943 \\
0.971\end{array}$ & $\begin{array}{l}0.881 \\
0.943\end{array}$ & $\begin{array}{l}0.951 \\
0.962\end{array}$ \\
\hline & Z (P value) & & & & & & & $-1.04(0$ & \\
\hline
\end{tabular}

Beside age and gender, Table 4 shows the percentage of any reported problem for each EQ-5D dimension, and the mean (SD) of EQ-VAS and EQ index scores by other socio-demographic characteristics.
Lower education indicated more problems in mobility, usual activities and more pain $\left(p<0.05\right.$, chi $^{2}$ test). Lower education also had lower EQ index score $(\mathrm{p}<$ 0.05 , one-way analysis of variance). Percentage of any 
Table 4 Percentage of a general population sample reporting any problem by dimension, EQ-VAS \& EQ index score by other demographic variables

\begin{tabular}{|c|c|c|c|c|c|c|c|}
\hline & Mobility & Self-care & Usual activities & Pain/discomfort & Anxiety/depression & EQ-VAS $(95 \% \mathrm{Cl})$ & EQ-index $(95 \% \mathrm{Cl})$ \\
\hline \multicolumn{8}{|l|}{ Highest education } \\
\hline Primary school \& lower $(n=138)$ & $10.9 \%$ & $1.4 \%$ & $9.4 \%$ & $37.0 \%$ & $25.4 \%$ & $84.8(82.9,86.8)$ & $0.943(0.924,0.961)$ \\
\hline High school $(n=867)$ & $5.4 \%$ & $0.9 \%$ & $4.1 \%$ & $30.3 \%$ & $24.6 \%$ & $86.2(85.5,87.0)$ & $0.959(0.954,0.963)$ \\
\hline College \& above $(n=291)$ & $3.8 \%$ & $1.0 \%$ & $3.4 \%$ & $25.1 \%$ & $34.4 \%$ & $85.9(84.7,87.0)$ & $0.959(0.952,0.965)$ \\
\hline$P$ value & 0.01 & 0.91 & 0.01 & 0.04 & 0.00 & 0.40 & 0.04 \\
\hline \multicolumn{8}{|l|}{ Employment status } \\
\hline Full time employee $(n=382)$ & $2.6 \%$ & $0 \%$ & $1.8 \%$ & $28.3 \%$ & $29.1 \%$ & $87.5(86.6,88.5)$ & $0.963(0.957,0.968)$ \\
\hline Part time \& freelancer $(n=451)$ & $4.2 \%$ & $0.7 \%$ & $4.7 \%$ & $29.3 \%$ & $26.6 \%$ & $85.6(84.5,86.7)$ & $0.960(0.955,0.966)$ \\
\hline $\operatorname{Retired}(n=240)$ & $13.3 \%$ & $2.9 \%$ & $7.1 \%$ & $37.5 \%$ & $16.7 \%$ & $83.8(82.2,85.5)$ & $0.948(0.937,0.958)$ \\
\hline Student $(n=132)$ & $1.5 \%$ & $0.8 \%$ & $3.0 \%$ & $17.4 \%$ & $40.1 \%$ & $88.7(87.3,90.0)$ & $0.964(0.957,0.972)$ \\
\hline Others $(n=91)$ & $11.0 \%$ & $3.3 \%$ & $11.0 \%$ & $37.4 \%$ & $26.4 \%$ & $83.7(80.7,86.8)$ & $0.930(0.902,0.957)$ \\
\hline$P$ value & 0.00 & 0.00 & 0.00 & 0.00 & 0.00 & 0.00 & 0.00 \\
\hline \multicolumn{8}{|l|}{ Health Insurance } \\
\hline With insurance $(n=1239)$ & $5.7 \%$ & $1.1 \%$ & $4.4 \%$ & $29.9 \%$ & $26.8 \%$ & $86.1(85.5,86.8)$ & $0.957(0.953,0.961)$ \\
\hline Without insurance $(n=57)$ & $3.5 \%$ & $0 \%$ & $7.0 \%$ & $29.8 \%$ & $28.1 \%$ & $82.8(79.4,86.1)$ & $0.953(0.933,0.974)$ \\
\hline$P$ value & 0.48 & 0.42 & 0.36 & 1.00 & 0.83 & 0.03 & 0.71 \\
\hline \multicolumn{8}{|l|}{ Residence of origin } \\
\hline $\operatorname{City}(n=757)$ & $6.7 \%$ & $1.1 \%$ & $4.1 \%$ & $31.2 \%$ & $23.7 \%$ & $85.6(84.8,86.5)$ & $0.957(0.952,0.962)$ \\
\hline County $(n=86)$ & $5.8 \%$ & $1.2 \%$ & $8.1 \%$ & $32.6 \%$ & $34.9 \%$ & $85.4(83.1,87.7)$ & $0.952(0.941,0.964)$ \\
\hline Township or village $(n=453)$ & $3.8 \%$ & $1.1 \%$ & $4.6 \%$ & $27.2 \%$ & $30.7 \%$ & $86.8(85.8,87.8)$ & $0.957(0.950,0.965)$ \\
\hline$P$ value & 0.09 & 0.99 & 0.23 & 0.29 & 0.00 & 0.19 & 0.82 \\
\hline \multicolumn{8}{|l|}{ Cities } \\
\hline Beijing & $3.0 \%$ & $0 \%$ & $2.3 \%$ & $28.2 \%$ & $17.9 \%$ & $88.5(87.4,89.7)$ & $0.968(0.962,0.974)$ \\
\hline Chengdu & $6.6 \%$ & $1.2 \%$ & $6.3 \%$ & $34.8 \%$ & $31.6 \%$ & $84.9(83.4,86.5)$ & $0.949(0.941,0.957)$ \\
\hline Guiyang & $7.7 \%$ & $1.2 \%$ & $5.8 \%$ & $21.8 \%$ & $28.0 \%$ & $86.0(84.7,87.2)$ & $0.959(0.949,0.969)$ \\
\hline Nanjing & $5.6 \%$ & $1.5 \%$ & $4.5 \%$ & $36.7 \%$ & $34.1 \%$ & $85.2(83.8,86.6)$ & $0.948(0.939,0.956)$ \\
\hline Shenyang & $5.2 \%$ & $1.6 \%$ & $4.0 \%$ & $27.6 \%$ & $22.4 \%$ & $85.4(83.8,87.0)$ & $0.961(0.952,0.969)$ \\
\hline$P$ value & 0.21 & 0.41 & 0.21 & 0.00 & 0.00 & 0.00 & 0.00 \\
\hline
\end{tabular}

reported problem all differed by employment status $\left(p<0.01, \mathrm{chi}^{2}\right.$ test), full time employees reported least problems with self-care and usual activities; students reported the least problems with mobility and less pain/discomfort; retired reported least anxiety/depression. Students reported the highest score in EQ-VAS and EQ index score. Percentage of reported problem in any dimensions did not differ between the insured respondents and respondents without insurance, but the EQ-VAS of the insured was higher than those without insurance $(p<0.05$, two-sample t-test). In terms of original place of residence, residents from the city reported less anxiety $\left(p<0.01, \mathrm{chi}^{2}\right.$ test). Difference were also found between cities in pain/discomfort, anxiety/depression, EQ-VAS and EQ index score.

Socio-demographic characteristics which significantly predicted any problems in EQ-5D dimensions, and EQVAS and EQ index scores, are reported in Table 5, where the reported problem in each dimension was reported as an odds ratio, and the EQ-VAS, EQ index scores were reported as regression coefficients. Notably, reported problems with anxiety/depression declined along age groups (odds ratio: 0.58 for $30-59$ years; 0.40 for $>=60$ years respectively). Males had 1.45 lower EQ-VAS value than females. All outcomes varied with employment status. For example, compared to the group with full time job, unemployed group reported 4.04 lower EQ-VAS value and 0.03 lower EQ-index score, retired group reported 3.93 lower EQ-VAS value and 0.02 lower EQ-index score. Respondents from the county were found more reported problem in usual activities (odds ratio: 2.58).

\section{Discussion}

This is the first EQ-5D-5L norms study from China. These general population-based norms provide insights into 
Table 5 The association between HRQoL data and demographic factors ( $N=1296)$

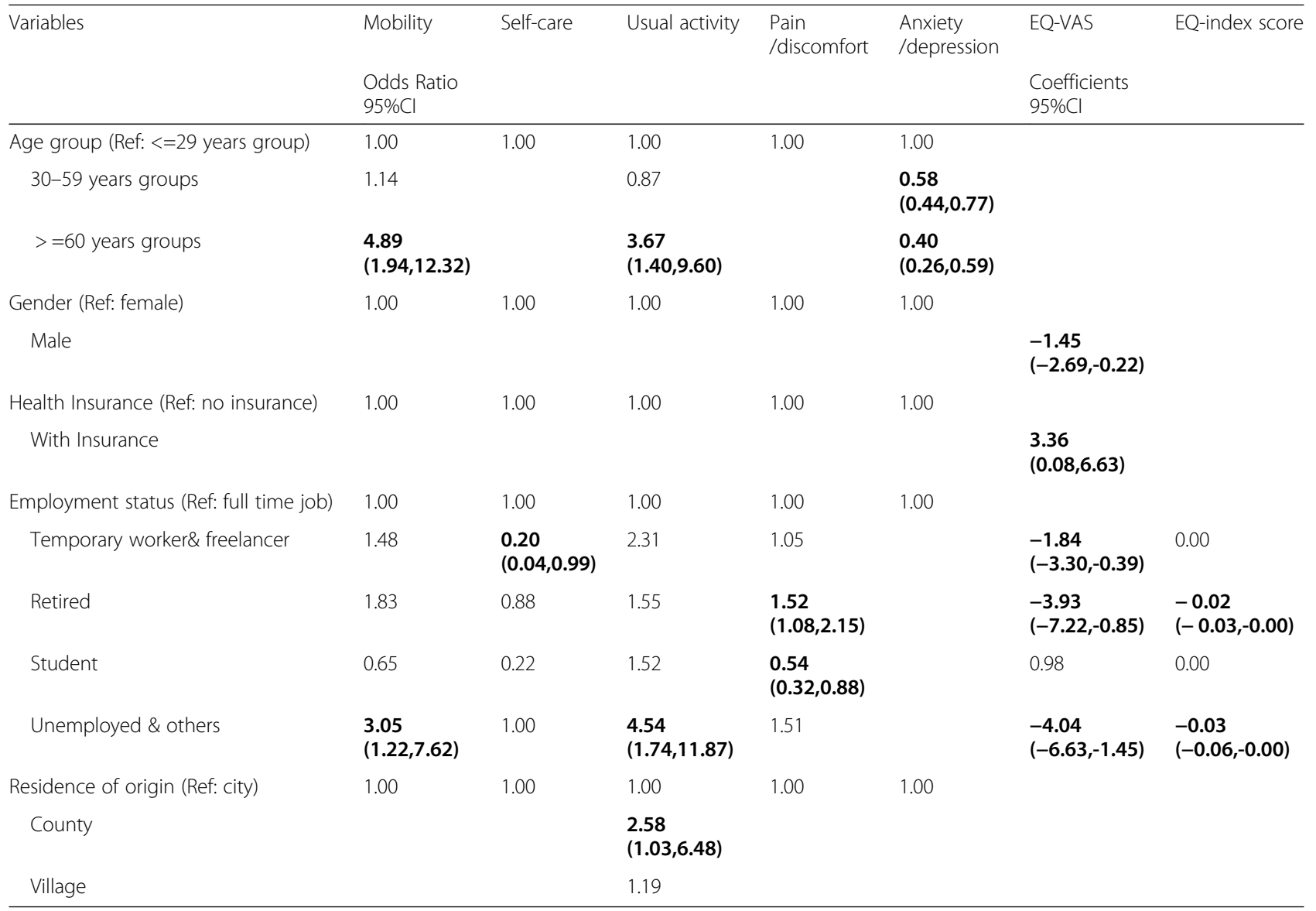

Note: Cl: Confidence interval, Bolded: statistically significant; $95 \% \mathrm{Cls}$ were calculated using robust standard error

HRQoL in China and how HRQoL varies between different socio-economic groups. More importantly, it facilitates interpretation of the cost effectiveness studies which use QALY as a health outcome. As HRQoL instruments measure postulated constructs, the set of norm scores provides a reference point to interpret an HRQoL study's results by comparing HRQoL between the general population and patients with specific conditions from similar age and gender groups $[19,20]$.

Compared to the Chinese EQ-5D-3L norms reported in 2008 [21], our study showed a significant increase in problems reported in the last two dimensions. This could be either because there were more problems in these two dimensions compared to the past, or that the five-level EQ-5D was more sensitive in identifying the mild problems in these dimensions. While it is not possible to detangle such change in our study, in several studies comparing norm scores between EQ-5D-3L and EQ-5D-5L, the researchers reported the $5 \mathrm{~L}$ questionnaire suffered less ceiling effect, had less standard deviation in the index value, and had wider spread of health states, which all suggests the improved sensitivity for the $5 \mathrm{~L}$ questionnaire $[4,13,16]$. HRQoL inequalities were shown in China between different socio-demographic groups and regions, based on previous research [21-24]. Such disparities were confirmed by our multivariable analysis, with lower socio-economic status related to lower HRQoL.

Some results from our study were in line with other countries' EQ-5D-5L norms [4, 6, 13-17, 25]: the first three dimensions of EQ-5D had less reported problems compared to the last two dimensions, with pain/discomfort being the most prevalent dimension; women reported lower EQ index score than men; EQ-VAS and EQ index score declined with age. Two differences were noted, first, in previous EQ-5D norms studies conducted in China and other countries, the percentage of reported problems in anxiety/depression increased with age $[1,4,6,13,25,26]$, our results suggest the opposite: the anxiety/depression problem was more prevalent in the younger population. One possible explanation is that the younger generation living in urbans areas perceived more psychological pressures than the older generation due to the fast-paced life in urban China. Second, females reported slightly higher EQ-VAS values 
than males, which is inconsistent with EQ-5D-3L norm values in China [21]: this discrepancy could be due to the difference in the two study samples' compositions. The EQ-VAS score is predicted by several demographic variables and in our study sample, females were in higher socio-economic groups.

One limitation of this study is that the sample was collected in five urban areas in China, which is not representative of the whole Chinese population. As socioeconomic differences exist between different areas, also between urban and rural areas in China, the health status of residents may differ by type of area [27]. Furthermore, most respondents were recruited in public locations, therefore the sample may have left out those who were not able to go outside. This may have led to a selection bias towards healthy respondents and underreported problems with mobility and usual activities. Nevertheless, we did not correct for this bias in our result as we did not know the exact proportion of respondents missed out in the sample. Third, this is a cross-sectional study, which provided insights into relationship between HRQoL data and socio-demographic variables. In terms of understanding the causal relationship between variables and controlling for unobserved heterogeneity, longitudinal data is needed [28-30].

\section{Conclusions}

This study has offered the first EQ-5D-5L urban population norms for China. Disparities exist in self-reported health status measured by EQ-5D-5L across socio-economic groups. Further research into rural HRQoL and into using a national representative sample is warranted.

\section{Acknowledgements}

We thank the EuroQol Group and Peking University to fund this study. We also thank Elly Stolk from the EuroQol Office for her constructive suggestions for the manuscript.

\section{Funding}

This study was cofunded by the EuroQol Group and the Peking University China Center for Health Economic Research.

\section{Availability of data and materials}

The datasets generated during and/or analyzed during the current study are available from the corresponding author on reasonable request.

\section{Authors' contributions}

Author GL proposed this study and prepared the data collection, author ZY drafted the manuscript and performed the data analysis, author NL supervised the data collection and edited the manuscript, author JB contributed to the data analysis and edited the manuscript. All authors read and approved the final manuscript.

\section{Ethics approval and consent to participate}

All procedures performed in studies involving human participants were in accordance with the ethical standards of Peking University and with the 1964 Helsinki declaration and its later amendments or comparable ethical standards. A waiver of the informed consent was approved as this study did not provide any intervention to participants. Participants can withdraw at any time without any consequences.
Consent for publication

All authors have agreed to publish this study.

\section{Competing interests}

Author $\mathrm{GL}$ declares that he has no conflict of interest. Authors $Z Y, N L$ and JB are members of the EuroQol Group.

\section{Publisher's Note}

Springer Nature remains neutral with regard to jurisdictional claims in published maps and institutional affiliations.

\section{Author details}

${ }^{1}$ Health Services Management Department, Guizhou Medical University, No.9 Beijing Road, Guiyang, China. ${ }^{2}$ Medical Psychology and Psychotherapy, Erasmus Medical Center, Wytemaweg, 80 Rotterdam, The Netherlands. ${ }^{3}$ National School of Development, Peking University, 5 Yiheyuan Road, Beijing 100871, China. ${ }^{4}$ Saw Swee Hock School of Public Health, National University of Singapore, 6 Medical Drive, Block MD3, Singapore 117597, Singapore.

Received: 9 March 2018 Accepted: 23 October 2018

Published online: 08 November 2018

\section{References}

1. Szende A, Janssen B, Cabases JM. Self-reported population health: an international perspective based on EQ-5D. New York: Springer; 2014.

2. Luo N, Li M, Liu GG, Lloyd A, de Charro F, Herdman M. Developing the Chinese version of the new 5-level EQ-5D descriptive system: the response scaling approach. Qual Life Res. 2013;22(4):885-90.

3. Luo N, Liu G, Li M, Guan H, Jin X, Rand-Hendriksen K. Estimating an EQ-5D5L value set for China. Value Health. 2017;20(4):662-9.

4. Feng Y, Devlin N, Herdman M. Assessing the health of the general population in England: how do the three- and five-level versions of EQ-5D compare? Health Qual Life Outcomes. 2015;13:171.

5. Janssen MF, Pickard AS, Golicki D, Gudex C, Niewada M, Scalone L, et al. Measurement properties of the EQ-5D-5L compared to the EQ5D-3L across eight patient groups: a multi-country study. Qual Life Res. 2013;22(7):1717-27

6. Kim TH, Jo MW, Lee SI, Kim SH, Chung SM. Psychometric properties of the EQ-5D-5L in the general population of South Korea. Qual Life Res. 2013; 22(8):2245-53.

7. Janssen MF, Bonsel GJ, Luo N. Is EQ-5D-5L better than EQ-5D-3L? A headto-head comparison of descriptive systems and value sets from seven countries. PharmacoEconomics. 2018:36(6):675-97.

8. Abdin E, Subramaniam M, Vaingankar JA, Luo N, Chong SA. Measuring health-related quality of life among adults in Singapore: population norms for the EQ-5D. Qual Life Res. 2013;22(10):2983-91.

9. Oppe M, Devlin NJ, van Hout B, Krabbe PFM, de Charro FA. Program of methodological research to arrive at the new international EQ-5D-5L valuation protocol. Value Health. 2014;17(4):445-53.

10. Jin XJ, Liu GG, Luo N, Li HC, Guan HJ. Xie F. is bad living better than good death? Impact of demographic and cultural factors on health state preference. Qual Life Res. 2016;25(4):979-86.

11. Oppe M, Rand-Hendriksen K, Shah K, Ramos-Goni JM, Luo N. EuroQol protocols for time trade-off valuation of health outcomes. PharmacoEconomics. 2016;34(10):993-1004.

12. Pan $\mathrm{CW}$, Sun $\mathrm{HP}$, Wang $X Z, \mathrm{Ma} Q \mathrm{QH}, \mathrm{Xu}$ Y, Luo N, et al. The EQ-5D-5L index score is more discriminative than the EQ-5D-3L index score in diabetes patients. Qual Life Res. 2015;24(7):1767-74.

13. Scalone L, Cortesi PA, Ciampichini R, Cesana G, Mantovani LG. Health Related Quality of Life norm data of the general population in Italy: results using the EQ-5D-3L and EQ-5D-5L instruments. Epidemiol Biostat Pu. 2015;12(3). https://doi.org/10.2427/11457.

14. McCaffrey N, Kaambwa B, Currow DC, Ratcliffe J. Health-related quality of life measured using the EQ-5D-5L: south Australian population norms. Health Qual Life. 2016;14:133.

15. Hinz A, Kohlmann T, Stobel-Richter Y, Zenger M, Brahler E. The quality of life questionnaire EQ-5D-5L: psychometric properties and normative values for the general German population. Qual Life Res. 2014;23(2):443-7. 
16. Shiroiwa T, Fukuda T, Ikeda S, Igarashi A, Noto S, Saito S, et al. Japanese population norms for preference-based measures: EQ-5D-3L, EQ-5D-5L, and SF-6D. Qual Life Res. 2016;25(3):707-19.

17. Garcia-Gordillo MA, Adsuar JC, Olivares PR. Normative values of EQ-5D-5L: in a Spanish representative population sample from Spanish health survey. 2011 Qual Life Res. 2016;25(5):1313-21.

18. Meng Q, Fang H, Liu X, Yuan B, Xu J. Consolidating the social health insurance schemes in China: towards an equitable and efficient health system. Lancet. 2015;386(10002):1484-92.

19. Fayers PM, Machin D. Quality of life: the assessment, analysis and interpretation of patient-reported outcomes. England: Wiley; 2007.

20. Rand-Hendriksen K, Ramos-Goni JM, Augestad LA, Luo N. Less is more: crossvalidation testing of simplified nonlinear regression model specifications for EQ-5D-5L health state values. Value Health. 2017;20(7):945-52.

21. Sun S, Chen J, Johannesson M, Kind P, Xu L, Zhang Y, et al. Regional differences in health status in China: population health-related quality of life results from the National Health Services Survey 2008. Health Place. 2011; 17(2):671-80.

22. Li H, Wei X, Ma A, Chung RY. Inequalities in health status among rural residents: EQ-5D findings from household survey China. Int J Equity Health. 2014;13:41.

23. Zhang T, Shi WX, Huang ZQ, Gao D, Guo ZY, Liu JY, et al. Influence of culture, residential segregation and socioeconomic development on rural elderly health-related quality of life in Guangxi, China. Health Qual Life. 2016;14:98.

24. Dong WL, Li YC, Wang ZQ, Jiang YY, Mao F, Qi L, et al. Self-rated health and health-related quality of life among Chinese residents, China, 2010. Health Qual Life. 2016;14:5.

25. Golicki D, Niewada M. EQ-5D-5L polish population norms. Arch Med Sci. 2015;13(1):191-200.

26. Sun $\mathrm{S}$, Chen J, Johannesson M, Kind P, Xu L, Zhang Y, et al. Population health status in China: EQ-5D results, by age, sex and socio-economic status, from the National Health Services Survey 2008. Qual Life Res. 2011; 20(3):309-20.

27. Liu GG, Wu H, Li M, Gao C, Luo N. Chinese time trade-off values for EQ-5D health states. Value Health. 2014;17(5):597-604.

28. Visser M, Verbaan D, van Rooden S, Marinus J, van Hilten J. Stiggelbout a. a longitudinal evaluation of health-related quality of life of patients with Parkinson's disease. Value Health. 2009:12(2):392-6.

29. Hajek A, Brettschneider C, Mallon T, Ernst A, Mamone S, Wiese B, et al. The impact of social engagement on health-related quality of life and depressive symptoms in old age - evidence from a multicenter prospective cohort study in Germany. Health Qual Life Outcomes. 2017;15(1):140.

30. Alva M, Gray A, Mihaylova B, Clarke P. The effect of diabetes complications on health-related quality of life: the importance of longitudinal data to address patient heterogeneity. Health Econ. 2014;23(4):487-500.

Ready to submit your research? Choose BMC and benefit from:

- fast, convenient online submission

- thorough peer review by experienced researchers in your field

- rapid publication on acceptance

- support for research data, including large and complex data types

- gold Open Access which fosters wider collaboration and increased citations

- maximum visibility for your research: over $100 \mathrm{M}$ website views per year

At $\mathrm{BMC}$, research is always in progress.

Learn more biomedcentral.com/submissions 